\title{
ON FIXED POINT THEOREM IN NON-ARCHIMEDEAN FUZZY NORMED SPACES
}

\author{
M.E. EGWE* \\ Department of Mathematics, University of Ibadan, Ibadan, Nigeria \\ ${ }^{*}$ Corresponding author: murphy.egwe@ui.edu.ng
}

\begin{abstract}
Let $(\mathfrak{X}, N)$ be a non-archimedean fuzzy normed space and $(\mathfrak{X},\|\cdot\|)$, a non-archimedean normed space where $\mathfrak{X}$ is a linear space over a linearly ordered non-archimedean field $\mathbb{K}$ with a non-archimedean valuation. We give a proof of the fixed point theorem in non-archimedean Fuzzy normed space.
\end{abstract}

\section{Introduction}

Definition 1.1 [9]: A valuation is a map $|\cdot|$ from a field $\mathbb{K}$ into a non-negative reals such that

(i) $|a|=0$ if and only if $a=0$

(ii) $|a b|=|a||b|$

(iii) $|a+b| \leq|a|+|b|$ for all $a, b \in \mathbb{K}$ (triangle inequality).

When a field $\mathbb{K}$ carries an absolute value $|\cdot|$, it is called a valued field $(\mathbb{K},|\cdot|)$. Examples of the pair $(\mathbb{K},|\cdot|)$ is called a valued field. Examples of valuations are provided by the usual absolute values of $\mathbb{R}$ and $\mathbb{C}$. In the definition above, if the triangle inequality is replaced by a strong triangle inequality, i.e, $|a+b| \leq \max (|a|,|b|)$ for all $a, b \in K$, the map || is then called a non-archimedean or ultrametric valuation.

Theorem 1.2 [1]: Let be a Complete space, $0<\lambda<1$, and $f: X \rightarrow X$ be a map such that $\|f(x)-f(y)\| \leq$ $\|x-y\|$ for all $x, y \in X$. Then there exists a unique point $x_{\circ}$ such that $f\left(x_{\circ}\right)=x_{\circ}$.

This fixed point in several cases have been obtained in non-archimedean normed and metric spaces (see [2], [4] [5], [6]). In this paper, we shall prove a version given in [4] for non-archimedean fuzzy normed spaces.

Received 2019-10-08; accepted 2019-11-14; published 2020-01-02.

2010 Mathematics Subject Classification. 46S10, 46S40, 47H10.

Key words and phrases. Fixed point, Non-archimedean, Fuzzy normed space, Spherically complete.

(C)2020 Authors retain the copyrights of their papers, and all open access articles are distributed under the terms of the Creative Commons Attribution License. 


\section{MAIN RESUlT}

Definition $2.1[4],[11]$ : Let $(\mathfrak{X},\|\cdot\|)$, a non-archimedean normed space. A function $N: \mathfrak{X} \times \mathbb{R} \rightarrow[0,1]$ is called a nonarhimedean norm on $\mathfrak{X}$ if for all $x, y \in \mathfrak{X}$ and all $s, t \in \mathbb{R}$,

(i) $N(x, t)=0$ for $t \leq 0$,

(ii) $N(x, t)=1$ if and only if $x=0$ for all $t>0$,

(iii) $N(\lambda x, t)=N\left(x, \frac{t}{|\lambda|}\right)$ for $\lambda \neq 0$

(iv) $N(x+y, \max \{s, t\}) \geq \min \{N(x, s), N(y, t)\}$

(v) $N(x, *)$ is nondecreasing function of $\mathbb{R}$ and $\lim _{t \rightarrow \infty} N(x, t)=1$

Definition 2.2: Let $(\mathfrak{X}, N)$ be a non-archimedean fuzzy normed space. A closed ball in $(\mathfrak{X}, N)$ with centre $a$ is the set of points $B(x, t):=\{N(x-a, t) \leq r$,$\} where t \in \mathbb{R}^{+}$.

Definition 2.3: A sequence of $n$-closed balls $B\left(x_{1}, t\right) \subseteq B\left(x_{2}, t\right) \subseteq \cdots \subset B\left(x_{n}, t\right)$ is called a sequence of closed balls ordered by inclusion.

Definition 2.4: Let $(\mathfrak{X}, N)$ be a non-archimedean fuzzy normed space. Let $\left\{B\left(x_{i}, t\right)\right\}^{n}$ be a sequence of closed balls ordered by inclusion. Then, $(\mathfrak{X}, N)$ is said to be spherically complete if the sequence of closed balls $\{B(x, t)\}^{n}$ satisfies the finite intersection property in $(\mathfrak{X}, N)$. i.e., $\bigcap_{i=1}^{n}\left\{B\left(x_{i}, t\right)\right\} \neq 0$.

Theorem 2.5 [1] Let $\mathfrak{V}$ be a complete normed linear space, $0<\alpha<1$, and $\varphi: \mathfrak{V} \rightarrow \mathfrak{V}$ such that $\|\varphi(u)-\varphi(\nu)\| \leq \alpha\|u-\nu\|$ for all $u, \nu \in \mathfrak{V}$. Then, there exists a fixed point, $u_{\circ} \in \mathfrak{V}$ such that $\varphi\left(u_{\circ}\right)=u_{\circ}$.

A version of this theorem on non-archimedean normed space was proved in [6] as seen in the next result.

Proposition 2.6 [6]: Suppose that $\mathfrak{X}$ and $\mathfrak{Y}$ are non-archimedean normed over a non-archimedean field $\mathbb{K}$ with $|p| \neq 1$ for some $p \in \mathbb{N}$. Assume that $\mathfrak{X}$ or $\mathfrak{Y}$ is spherically complete. If $f: \mathfrak{X} \rightarrow \mathfrak{Y}$ is a surjective isometry, then for each $x \in \mathfrak{X}$, there exists a unique $y \in \mathfrak{X}$ such that $f(x)+f(y)=f\left(\frac{x+y}{p}\right)$.

We now state and prove a version of this result for the non-archimedean fuzzy normed spaces

Proposition 2.7: Let $(\mathfrak{X}, N)$ and $(\mathfrak{Y}, N)$ be non-archimedean fuzzy normed spaces over a non-archimedean field $\mathbb{K}$ with $|p|>1$ for some $p \in \mathbb{N}$. Assume that $\mathfrak{X}$ or $\mathfrak{Y}$ is spherically complete. If $f:(\mathfrak{X}, N) \rightarrow(\mathfrak{Y}, N)$ is a surjective isometry, then for each $u \in \mathfrak{X}$, there exists a unique $\nu \in \mathfrak{X}$ such that $f(u)+f(\nu)=f\left(\frac{u+\nu}{p}\right)$. Proof: First, we prove that $(\mathfrak{X}, N)$ or $(\mathfrak{Y}, N)$ is spherically complete. Suppose that $(\mathfrak{X}, N)$ is spherically complete and let $\left\{B\left(y_{i}, t\right)\right\}^{n}$ be a sequence od closed balls in (YY, $\left.N\right)$ ordered by inclusion. Then by the surjectivity of $f$, there is a sequence $\left\{B\left(x_{i}, t\right)\right\}^{n}$ of closed balls in $(\mathfrak{X}, N)$ ordered by inclusion with

$$
B\left(x_{1}, t\right)=f^{-1}\left(B\left(y_{1}, t\right)\right) \subseteq B\left(x_{2}, t\right)=f^{-1}\left(B\left(y_{2}, t\right)\right) \subseteq \cdots \subseteq B\left(x_{n}, t\right)=f^{-1}\left(B\left(y_{n}, t\right)\right) .
$$

Thus,

$$
\bigcap^{n} f^{-1}\left(B\left(y_{i}, t\right)\right) \neq \phi \text { as } \phi \neq \bigcap^{n}\left(B\left(x_{i}, t\right)\right)=f^{-1}\left(B\left(y_{i}, t\right)\right)
$$

because $(\mathfrak{X}, N)$ is spherically complete. Thus, $(\mathfrak{Y}, N)$ is spherically complete if $(\mathfrak{X}, N)$ is spherically complete. 
Conversely, let $(\mathfrak{Y}, N)$ be spherically complete and $\{B(x, t)\}^{n}$ a sequence of closed balls in $(\mathfrak{X}, N)$ ordered by inclusion. Then there exists a sequence $\left\{f\left(B\left(x_{i}, t\right)\right)\right\}^{n}$ of closed balls in $(\mathfrak{Y}, N)$ such that

$$
f\left(B\left(x_{1}, t\right)\right) \subseteq f\left(B\left(x_{2}, t\right)\right) \subseteq \cdots f\left(B\left(x_{n}, t\right)\right) .
$$

Then,

$$
B\left(x_{1}, t\right)=f\left(B\left(x_{1}, t\right)\right) \subseteq f\left(B\left(x_{2}, t\right)\right)=B\left(x_{2}, t\right) \subseteq \cdots f\left(B\left(x_{n}, t\right)\right)=B\left(x_{n}, t\right)
$$

and

$$
\bigcap^{n} B\left(x_{i}, t\right) \neq \phi \text { as } \phi \neq \bigcap^{n} f\left(B\left(x_{i}, t\right)\right)=\bigcap^{n}\left(B\left(x_{i}, t\right)\right)
$$

because $(\mathfrak{Y}, N)$ is spherically complete. Thus, $(\mathfrak{X}, N)$ is spherically complete if $(\mathfrak{Y}, N)$ is. This implies that $(\mathfrak{X}, N)$ or $(\mathfrak{Y}, N)$ is spherically complete.

Next, we show that there exists a unique $\nu \in \mathfrak{X}$ such that $f(u)+f(\nu)=f\left(\frac{u+\nu}{p}\right)$ for each $u \in \mathfrak{X}$. To do this,let $u \in \mathfrak{X}$, and consider the mapping $\varphi: \mathfrak{X} \rightarrow \mathfrak{X}: x \mapsto p x-u$. Now,

$$
\begin{aligned}
N(\varphi(x)-\varphi(y), t) & =N(p x-u-(p y-u), t) \\
& =N(p x-u-p y+u), t) \\
& =N((p x-p y), t) \\
& =N\left(x-y, \frac{t}{|p|}\right) \\
& <N(x-y, t) .
\end{aligned}
$$

Thus, there exists $M>1$ such that $M . N(\varphi(x)-\varphi(y), t)<N(x-y, t)$, i.e.,

$$
N(\varphi(x)-\varphi(y), t)<\frac{1}{M} N(x-y, t) .
$$

Obviously, $0<\frac{1}{M}<1$, and $N(\varphi(x)-\varphi(y), t) \leq N(x-y, t)$ which implies that $\varphi$ is a contractive mapping. Let $\psi: \mathfrak{Y} \rightarrow \mathfrak{Y}$ be an isometry defined by $\psi(y)=f(u)+y$. If $h=\varphi f^{-1} \psi f$. Then,

$$
\begin{aligned}
N(\varphi h(x)-h(y), t) & =N\left(\left(\varphi f^{-1} \psi f\right)(x)-\left(\varphi f^{-1} \psi f\right)(y), t\right) \\
& =N\left(p\left(\varphi f^{-1} \psi f\right)(x)-u-\left(p\left(\varphi f^{-1} \psi f\right)(y)\right), t\right) \\
& =N\left(p\left(\varphi f^{-1} \psi f\right)(x)-u-p\left(\varphi f^{-1} \psi f\right)(y)+u, t\right) \\
& =N\left(p\left(\varphi f^{-1} \psi f\right)(x)-p\left(\varphi f^{-1} \psi f\right)(y), t\right) \\
& =N\left(\left(\varphi f^{-1} \psi f\right)(x)-\left(\varphi f^{-1} \psi f\right)(y), \frac{t}{|p|}\right) \\
& <N\left(\left(\varphi f^{-1} \psi f\right)(x)-\left(\varphi f^{-1} \psi f\right)(y), t\right) \\
& =N((\psi f)(x)-(\psi f)(y), t) \\
& =N(f(x)-f(y), t) \\
& =N(x-y, t) .
\end{aligned}
$$

Similarly, there exists $K^{*}>1$ such that $K^{*} . N(h(x)-h(y), t) \leq N(x-y, t)$. This also implies that

$$
N(h(x)-h(y), t) \leq \frac{1}{K^{*}} N(x-y, t) .
$$


Since $\frac{1}{K^{*}}<1$ and $N(h(x)-h(y), t) \leq N(x-y, t)$, then, $h$ is a contraction mapping. By the fixed point theorem, $h$ has a unique fixed point $\nu$ such that

$$
\begin{aligned}
\left(\psi f^{-1} \psi f\right)(\nu) & =h(\nu) \\
& =\nu
\end{aligned}
$$

But

$$
\begin{aligned}
\psi\left(\frac{u+\nu}{p}\right) & =p \cdot \frac{u+\nu}{p}-u \\
& =u+\nu-u \\
& =\nu
\end{aligned}
$$

Therefore, $\psi(\nu)=\psi(f(\nu))=\psi\left(\frac{u+\nu}{p}\right)=f\left(\frac{u+\nu}{p}\right)$, as $f$, and $\psi$ are injections. Since $\psi(f(\nu))=$ $f(u)+f(\nu)$ by definition, it follows that $f(u)+f(\nu)=f\left(\frac{u+\nu}{p}\right)$.

Remark 2.8: It is necessary for $|p|>1$ for

(i) if $|p|>1$, then

$$
N(\varphi(u)-\varphi(\nu), p)=N(u-\nu, t)
$$

as

$$
N\left(u-\nu, \frac{t}{|p|}\right)=N(u-\nu, t)
$$

Also,

$$
N(h(u)-h(\nu), t)=N(u-\nu, t)
$$

as

$$
N\left(\left(f^{-1} \psi f\right)(u)-\left(f^{-1} \psi f\right)(\nu), \frac{t}{|p|}\right)=N\left(\left(f^{-1} \psi f\right)(u)-\left(f^{-1} \psi f\right)(\nu), t\right) .
$$

So, $\varphi$ and $h$ are not contraction mappings.

(ii) if $|p|=0$, then $p=0$ by definition of valuation. This negates the assumption that $p \in \mathbb{N}$.

Conflicts of Interest: The author(s) declare that there are no conflicts of interest regarding the publication of this paper.

\section{REFERENCES}

[1] D. Burago, Y. Burago, S. Ivanon: A course in Metric Geometry, Amer. Math. Soc. 2001.

[2] Y. Je Cho, T.M. Rassias, R. Saadati: Fuzzy Operator Theory in Mathematical Analysis. Springer International Publishing, 2018

[3] A. Granas, J. Dugunji: Fixed Point Theory, Springer, 2003.

[4] D. Kangb, H. Kohb, I.G. Chao: On the Mazur-Ulam theorem in non-archimedean fuzzy normed spaces, Appl. Math. Lett. 25(2012), 301-304.

[5] H. Mamghaderi, H.P. Masiha: On Stationary Points of Multivalued Strongly Contractive Mappings in Partially Ordered Ultrametric Spaces and non-Archimedean Normed Spaces. p-Adic Numbers, Ultr. Anal. Appl. 9(2)(2017), 144-150. 
[6] M.S. Moslehian, G. Sadeghi: A Mazur-Ulam theorem in non-archimedean normed spaces, Nonlinear Anal., Theory Methods Appl. 69(2008), 3405-3408.

[7] A. Narayanan, S. Vijayabalaji: Fuzzy n-normed linear spaces. Int. J. Math. Math. Sci. 24(2005), 3963-3977.

[8] C. Petalas, T. Vidalis: A fixed point theorem in non-archimedean vector spaces,Proc. Amer. Math. Soc. 118(3)(1993), 819-821.

[9] A.C.M. Rooij: Non-Archimedean functional Analysis, Marcel Dekker NY, 1978.

[10] F.Shi, C. Huang: Fuzzy bases and the fuzzy dimension of fuzzy vector spaces, Math. Commun. 15(2010), 303-310.

[11] Z. Wang, P.K. Sahoo: Stability of an ACQ-functional equation in various Stability of an ACQ-functional equation in various. J. Nonlinear Sci. Appl. 8(2015), 64-85. 